\title{
Mitigation of envelope instability through fast acceleration in linear accelerators
}

\author{
Ji Qiang* \\ Lawrence Berkeley National Laboratory, Berkeley, California 94720, USA
}

(Received 18 June 2018; published 26 November 2018)

\begin{abstract}
The space-charge driven envelope instability can be of great danger in high intensity accelerators. Linear accelerators were designed to avoid this instability by keeping the zero current phase advance per lattice period below 90 degrees. In this paper, we studied the acceleration effects on the instability in a periodic solenoid and radio-frequency (rf) focusing channel and a periodic quadrupole and rf focusing channel using a three-dimensional envelope model and self-consistent macroparticle simulations. Our results suggest that the envelope instability might be dramatically mitigated with a reasonable accelerating gradient in both channels. This suggests that the zero current phase advance without acceleration might be above 90 degrees in linear accelerators where the accelerating gradient is sufficiently high.
\end{abstract}

DOI: 10.1103/PhysRevAccelBeams.21.114201

\section{INTRODUCTION}

In a periodic accelerator system, when the zero current phase advance through one lattice period is above 90 degrees, the envelope oscillation of a charged particle beam with finite current can become unstable [1,2]. Such an envelope instability driven by the space-charge effects causes beam size blow up and potential particle loss inside the accelerator. In order to avoid the envelope instability, linear accelerators were designed with zero current phase advance per lattice period below 90 degrees [3-9]. However, the linear accelerator is not a perfect periodic system. In addition to the machine imperfections such as field amplitude error and misalignment error, the use of rf cavity acceleration breaks the longitudinal periodicity of the accelerator. The presence of rf acceleration helps damp the envelope oscillation and also modifies the transverse and longitudinal focusing strengths and space-charge forces. Previous studies have shown that resonances in circular accelerators can be crossed without significantly affecting the beam quality if the crossing speed is sufficiently fast [10-13]. With the use of superconducting rf cavity in the linear accelerator, the high accelerating gradient from the cavity may help overcome the envelope instability. In this paper, we report on mitigating strong envelope instability of a proton beam through a solenoid-rf lattice and through a quadrupole-rf

\footnotetext{
*jqiang@lbl.gov
}

Published by the American Physical Society under the terms of the Creative Commons Attribution 4.0 International license. Further distribution of this work must maintain attribution to the author(s) and the published article's title, journal citation, and DOI. lattice with 120 degree zero current transverse and longitudinal phase advances.

The envelope instability in a periodic transport channel has been extensively studied theoretically and experimentally since the 1980s [1,2,14-31]. (Some of those studies were summarized in a recently published monograph [32].) It is the lowest order (second-order) collective mode instability driven by the direct space-charge effects and could present great danger in high intensity accelerator operation. Recently, an analysis of bunched beam stability in a periodic solenoid-rf and in a periodic quadrupole-rf transport channel was done using a three-dimensional (3D) envelope model without acceleration [33]. A number of instability stopbands were identified in that study when the zero current phase advance per lattice period is beyond 90 degrees. In this paper, we study the effects of acceleration on the instability of envelope oscillation in these two types of focusing channels.

The organization of this paper is as follows: after the introduction, we present the 3D envelope instability analysis with acceleration in Sec. II; we present the study of mitigation of the envelope instability in a transverse solenoid and longitudinal if focusing and accelerating channel in Sec. III; we present the study of mitigation of the instability in a transverse quadrupole and longitudinal rf focusing and accelerating channel in Sec. IV; and draw conclusions in Sec. V.

\section{THREE-DIMENSIONAL ENVELOPE INSTABILITY MODEL WITH ACCELERATION}

In a linear accelerator, for a 3D uniform density ellipsoidal beam, the 3D envelope equations including acceleration are given as [34,35]: 


$$
\begin{aligned}
& \frac{d^{2} X}{d s^{2}}+\left(\frac{p_{0}^{\prime}}{p_{0}}\right) \frac{d X}{d s}+k_{x}^{2}(s) X-\frac{K u_{0} \pi \lambda_{3}}{l^{2}} G_{311}\left(X, Y, u_{0} T\right) X \\
& -\left(\frac{\delta}{l p_{0}}\right)^{2} \frac{\epsilon_{x}^{2}}{X^{3}}=0 \\
& \frac{d^{2} Y}{d s^{2}}+\left(\frac{p_{0}^{\prime}}{p_{0}}\right) \frac{d Y}{d s}+k_{y}^{2}(s) Y-\frac{K u_{0} \pi \lambda_{3}}{l^{2}} G_{131}\left(X, Y, u_{0} T\right) Y \\
& -\left(\frac{\delta}{l p_{0}}\right)^{2} \frac{\epsilon_{y}^{2}}{Y^{3}}=0 \\
& \frac{d^{2} T}{d s^{2}}+3\left(\frac{p_{0}^{\prime}}{p_{0}}\right) \frac{d T}{d s}+k_{t}^{2}(s) T-\frac{K u_{0} \pi \lambda_{3}}{l^{2}} G_{113}\left(X, Y, u_{0} T\right) T \\
& -\left(\frac{\delta}{l p_{0} u_{0}^{2}}\right)^{2} \frac{\epsilon_{t}^{2}}{T^{3}}=0
\end{aligned}
$$

with

$G_{l m n}(x, y, z)=\frac{3}{2} \int_{0}^{\infty} \frac{d s}{\left(x^{2}+s\right)^{l / 2}\left(y^{2}+s\right)^{m / 2}\left(z^{2}+s\right)^{n / 2}}$

where $X$ and $Y$ are horizontal and vertical rms beam sizes normalized by the scaling length $l=c / \omega, T$ is the rms longitudinal phase $(T=\omega \Delta t), \Delta t$ denotes the rms time of flight difference between the individual particle and the reference particle, $c$ is the speed of light in vacuum, $\omega$ is the rf angular frequency, $p_{0}=m c \gamma_{0} \beta_{0}$ is the reference particle momentum, the prime denotes derivative with respect to distance $s, k_{x}^{2}, k_{y}^{2}, k_{t}^{2}$ represent the external focusing forces $\left(\left(k_{x}(s)=k_{y}(s)=q B(s) /\left(2 p_{0}\right)\right.\right.$ for solenoids and $k_{x, y}^{2}(s)=$ $\pm q G(s) / p_{0}$ for quadrupoles, where $B$ is the solenoid root mean-squared magnetic field along the axis and $G$ is the quadrupole gradient, $k_{t}^{2}=\omega q E_{0} T_{t r} \sin \left(-\phi_{s}\right) /\left(m c^{3} \beta_{0}^{3} \gamma_{0}^{3}\right)$ for longitudinal rf focusing [36]), $u_{0}=\gamma_{0} \beta_{0}, \delta=m c, \epsilon_{x}$, $\epsilon_{y}$, and $\epsilon_{t}$ are normalized rms emittances, and $K$ is the generalized perveance associated with the space-charge strength given by:

$$
K=\frac{q I}{2 \pi \epsilon_{0} p_{0} v_{0}^{2} \gamma_{0}^{2}}
$$

where $I$ is the average current of the beam, $q$ is the charge of the particle, $\epsilon_{0}$ is the vacuum permittivity, $v_{0}$ is the speed of the reference particle, and $\gamma_{0}$ is the relativistic factor of the reference particle. The quantity $\lambda_{3}$ is a constant depending on the distribution of the beam. It was pointed out in Ref. [34] that the space-charge form factor $\lambda_{3}=1 / 5 \sqrt{5}$ for a uniform distribution depends only weakly on the type of distributions and is $1.01 / 5 \sqrt{5}$ for a parabolic distribution and $1.05 / 5 \sqrt{5}$ for a Gaussian distribution. In this study, we assume that the rf cavity design phase is fixed and the reference particle energy increases linearly inside the cavity due to the rf acceleration. The energy dependency of the external focusing and the space-charge effects is automatically included in the calculation through the changes of $\gamma_{0}$ and $\beta_{0}$ from the acceleration.

The above envelope equations can be linearized with respect to matched solutions as:

$$
\begin{aligned}
& X(s)=X_{0}(s)+x(s) \\
& Y(s)=Y_{0}(s)+y(s) \\
& T(s)=T_{0}(s)+t(s)
\end{aligned}
$$

where $X_{0}, Y_{0}$ and $Z_{0}$ denote the matched envelope solutions and $x, y$ and $t$ denote small perturbations

$x(s) \ll X_{0}(s), \quad y(s) \ll Y_{0}(s), \quad t(s) \ll T_{0}(s)$.

Let $\xi=\left(x, p_{x}, y, p_{y}, t, p_{t}\right)^{T}$, the equations of motion for the perturbations are given by:

$$
\frac{d \xi}{d s}=A_{6}(s) \xi(s)
$$

with the matrix $A_{6}$ given by:

$A_{6}(s)=\left(\begin{array}{cccccc}0 & \frac{\delta}{l p_{0}(s)} & 0 & 0 & 0 & 0 \\ a_{21}(s) & 0 & a_{23}(s) & 0 & a_{25}(s) & 0 \\ 0 & 0 & 0 & \frac{\delta}{l p_{0}(s)} & 0 & 0 \\ a_{23}(s) & 0 & a_{43}(s) & 0 & a_{45}(s) & 0 \\ 0 & 0 & 0 & 0 & 0 & \frac{\delta}{l p_{0}(s) u_{0}^{2}(s)} \\ a_{25}(s) & 0 & a_{45}(s) & 0 & a_{65}(s) & 0\end{array}\right)$

where

$a_{21}(s)=-\frac{l p_{0}}{\delta} k_{x}^{2}-3 \frac{\delta}{l p_{0}} \frac{\epsilon_{x}^{2}}{X_{0}^{4}}-\frac{K u_{0}^{2} \pi \lambda_{3}}{l}\left(3 X_{0}^{2} G_{511}-G_{311}\right)$

$$
\begin{gathered}
a_{23}(s)=-\frac{K u_{0}^{2} \pi \lambda_{3}}{l} X_{0} Y_{0} G_{331} \\
a_{25}(s)=-\frac{K u_{0}^{4} \pi \lambda_{3}}{l} X_{0} T_{0} G_{313} \\
a_{43}(s)=-\frac{l p_{0}}{\delta} k_{y}^{2}-3 \frac{\delta}{l p_{0}} \frac{\epsilon_{y}^{2}}{Y_{0}^{4}}-\frac{K u_{0}^{2} \pi \lambda_{3}}{l}\left(3 Y_{0}^{2} G_{151}-G_{131}\right) \\
a_{45}(s)=-\frac{K u_{0}^{4} \pi \lambda_{3}}{l} Y_{0} T_{0} G_{133}
\end{gathered}
$$




$$
\begin{aligned}
a_{65}(s)= & -\frac{l p_{0} u_{0}^{2}}{\delta} k_{z}^{2}-3 \frac{\delta}{l p_{0} u_{0}^{2}} \frac{\epsilon_{t}}{T_{0}^{4}}-\frac{K u_{0}^{4} \pi \lambda_{3}}{l} \\
& \times\left(3 u_{0}^{2} T_{0}^{2} G_{115}-G_{113}\right) .
\end{aligned}
$$

Let solution $\xi(s)=M_{6}(s) \xi(0)$, substituting this equation into Eq. (10) results in

$$
\frac{d M_{6}(s)}{d s}=A_{6}(s) M_{6}(s)
$$

where $M_{6}(s)$ denotes the $6 \times 6$ transfer matrix solution of $\xi(s)$ and $M_{6}(0)$ is a $6 \times 6$ unit matrix. The above ordinary differential equation can be solved using the matched envelope solutions and numerical integration. The stability of these envelope perturbations is determined by the eigenvalues of the transfer matrix $M_{6}(L)$ through one lattice period. For the envelope oscillation to be stable, all six eigenvalues (three pairs) of the $M_{6}(L)$ have to stay on the unit circle. The amplitude of the eigenvalue gives the envelope mode growth (or damping) rate through one lattice period, while the phase of the eigenvalue yields the mode oscillation frequency. When the amplitude of any eigenvalue is greater than one, the envelope oscillation becomes unstable.

\section{MITIGATION OF THE ENVELOPE INSTABILITY IN A SOLENOID AND RF CHANNEL}

We first studied the mitigation of the envelope instability in a transverse solenoid focusing and longitudinal $\mathrm{rf}$ accelerating and focusing channel. A schematic plot of this channel is shown in Fig. 1. Each period of the accelerator lattice consists of a 0.1 meter solenoid, a 0.1 meter drift, a 0.4 meter rf cavity, and 0.1 meter drift. The proton bunch has an initial kinetic energy of $10 \mathrm{MeV}$ and normalized rms emittances of $0.2 \mathrm{um}$ in all three directions. Figure 2 shows the envelope mode growth rate amplitudes as a function of depressed transverse phase advance inside the above lattice with 120 degree zero current transverse and 120 degree zero current longitudinal phase advances without and with $8 \mathrm{MV} / \mathrm{m}$ accelerating gradient inside the rf cavities. Here, the smaller depressed phase advance corresponds to the stronger space-charge defocusing effects and the higher proton beam bunch intensity. The depressed phase advances with acceleration

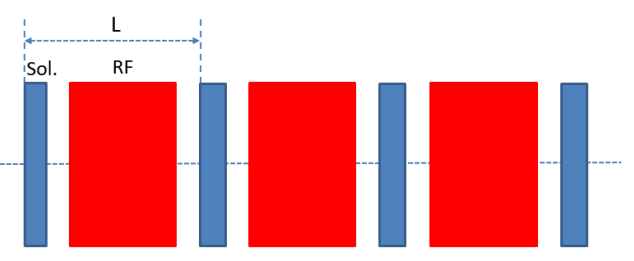

FIG. 1. Schematic plot of a periodic solenoid and rf channel. were calculated using the same lattice as those without acceleration. These phase advances include the energy dependency of the external focusing and the space-charge effects due to the acceleration. Without acceleration, the beam shows strong envelope instability. There are three instability stopbands below about 80 degree depressed transverse phase advances. The stopband below 65 degree depressed tune is due to the confluent resonance between two envelope oscillation modes. The other two stopbands are due to the half-integer parametric resonance between the focusing lattice and the envelope oscillation modes. With the presence of acceleration, the proton beam energy increases linearly inside the cavity with $8 \mathrm{MV} / \mathrm{m}$ accelerating gradient. The above envelope instability stopbands are significantly modified. The width of stopband is reduced from about 80 degrees to about 50 degrees. The instability growth rate maximum amplitude is also reduced from nearly 1.4 to about 1.2 .

In the above example, with acceleration, the envelope instability analysis is an approximate model due to the periodic assumption in the analysis even though the beam energy increases through a lattice period in the calculation of the transfer matrix. In a real lattice with acceleration, the proton beam energy will keep on increasing through the entire lattice. In order to study the effects of acceleration on the envelope instability in the real lattice, we carried out self-consistent macroparticle simulations using a 3D particle-in-cell code, IMPACT $[37,38]$. In the self-consistent simulation, a number of macroparticles were tracked through the lattice subject to the external focusing and accelerating and the space-charge forces. The parameters of external focusing elements were determined from the zero current phase advances without acceleration (same as those used in the envelope analysis). With the acceleration, both the external focusing and the space-charge forces vary automatically during the simulation depending on the beam energy. Here, we assumed a linear increase of beam energy inside the rf cavities from the acceleration.

We first selected an unstable point inside the depressed phase advance stopband with large instability growth rate. The transverse depressed phase advance for this point is 40 degrees, which represents a strong space-charge tune depression ratio of 0.33 . Here the tune depression ratio is defined as the ratio of the depressed phase advance to the zero current phase advance per lattice period. It is a measure of relative space-charge effects with respect to the external focusing. The smaller tune depression ratio, the stronger space-charge effects would be. We assumed $10 \%$ mismatch in all three directions of an initial Waterbag distribution and used about 625,000 macroparticles and $64 \times 64 \times 64$ grid points in the simulations. Figure 3 shows the horizontal and longitudinal rms size (normalized by the matched rms size) evolution and the emittance growth (emittance change divided by the initial emittance) evolution through the above lattice without and with $8 \mathrm{MV} / \mathrm{m}$ accelerating gradient in 

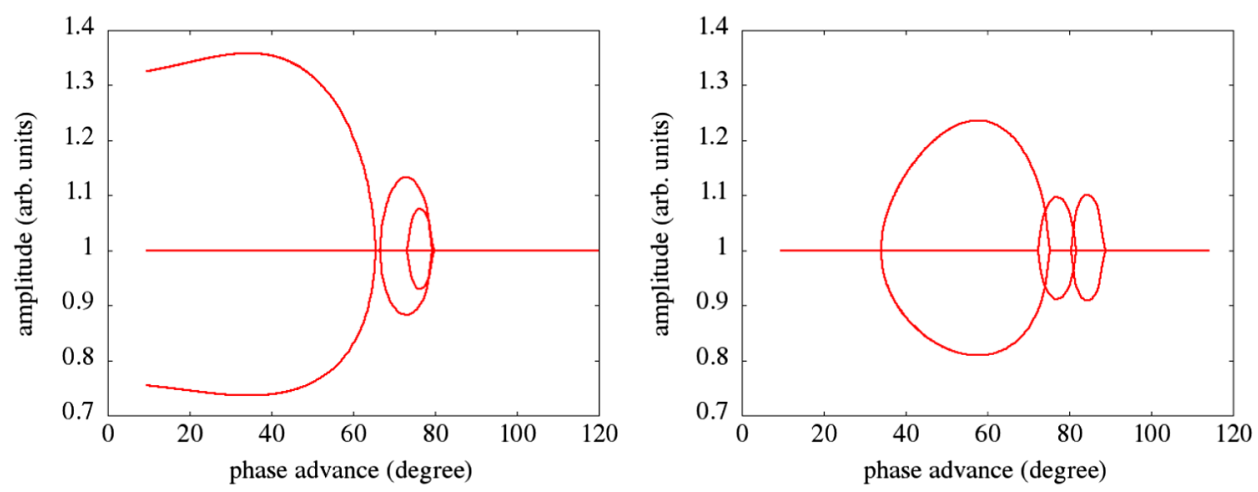

FIG. 2. The 3D envelope mode growth rate amplitudes as a function of depressed transverse phase advance with 120 degree zero current transverse and longitudinal phase advances in the periodic solenoid-rf channel without acceleration (left) and with $8 \mathrm{MV} / \mathrm{m}$ accelerating gradient (right).
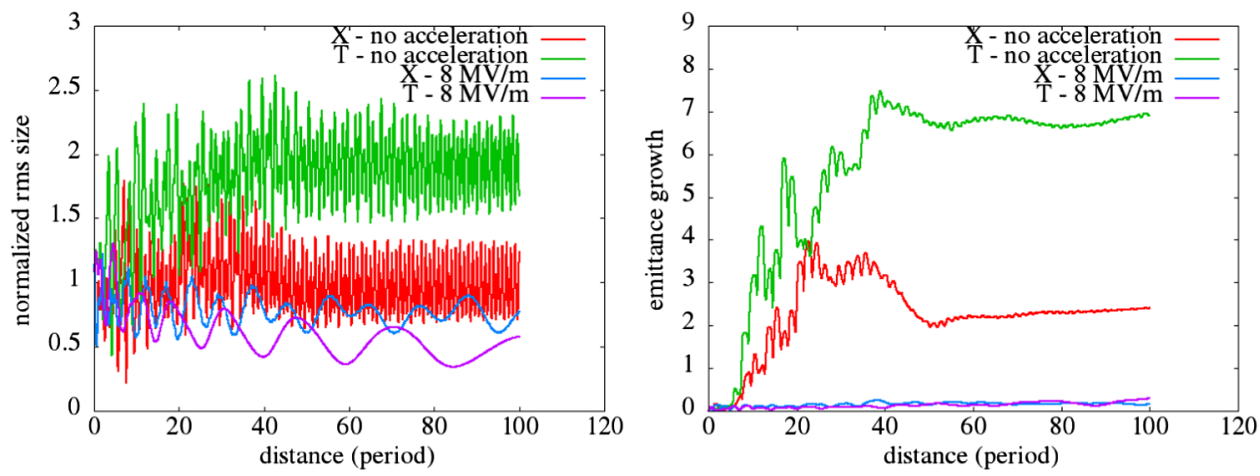

FIG. 3. The horizontal and longitudinal normalized rms size evolution (left) and emittance growth evolution (right) inside the solenoid-rf channel without acceleration and with acceleration. The depressed transverse phase advance is 40 degrees with 120 degree zero current transverse and longitudinal phase advances.
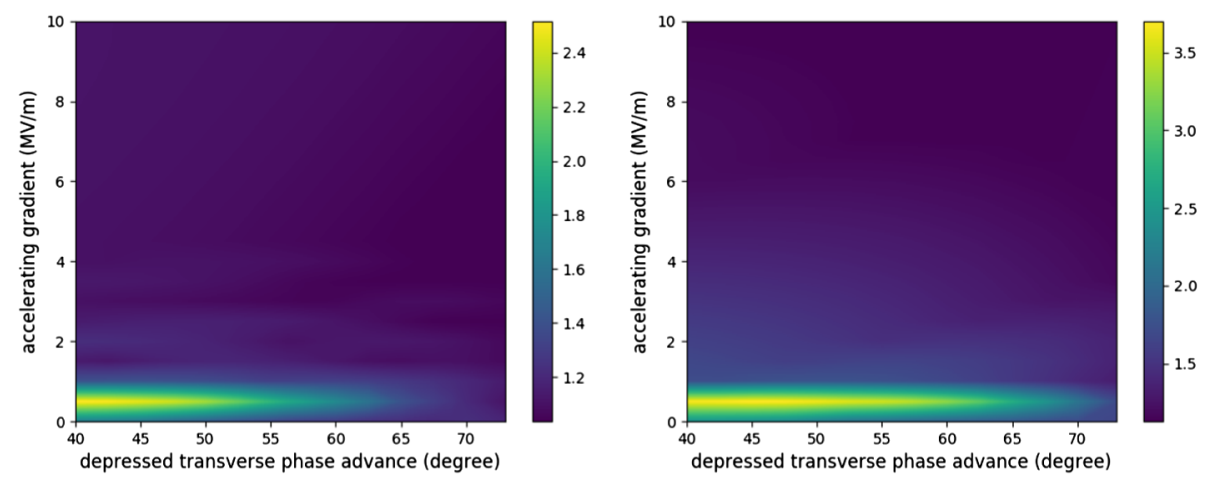

FIG. 4. The maximum normalized horizontal rms amplitude (left) and longitudinal rms amplitude (right) within 100 lattice periods as a function of accelerating gradient and depressed transverse phase advance in the solenoid-rf channel.

the $\mathrm{rf}$ cavities. Without acceleration, the transverse and longitudinal rms sizes grow quickly up to about 40 periods due to the instability. With the acceleration, the mismatched rms envelope oscillations growth is significantly damped and the beam becomes stable. Without acceleration, the envelope instability also causes large (more than a factor of 3 and 7) emittance growth. With the acceleration, the emittance growth is small and below 30\% through this lattice.

To see the effects from acceleration systematically, we calculated the maximum horizontal and longitudinal rms envelope amplitudes (normalized by the corresponding initial matched rms sizes) within 100 lattice periods and 

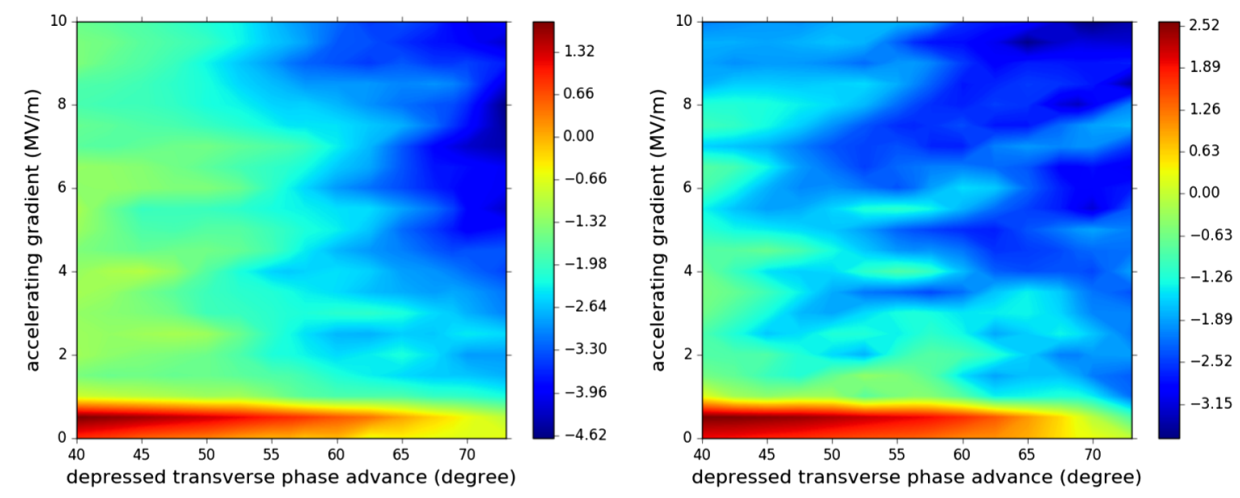

FIG. 5. The final log horizontal emittance growth (left) and longitudinal emittance growth (right) at the end of 100 lattice periods as a function of accelerating gradient and depressed transverse phase advance in the solenoid-rf channel.
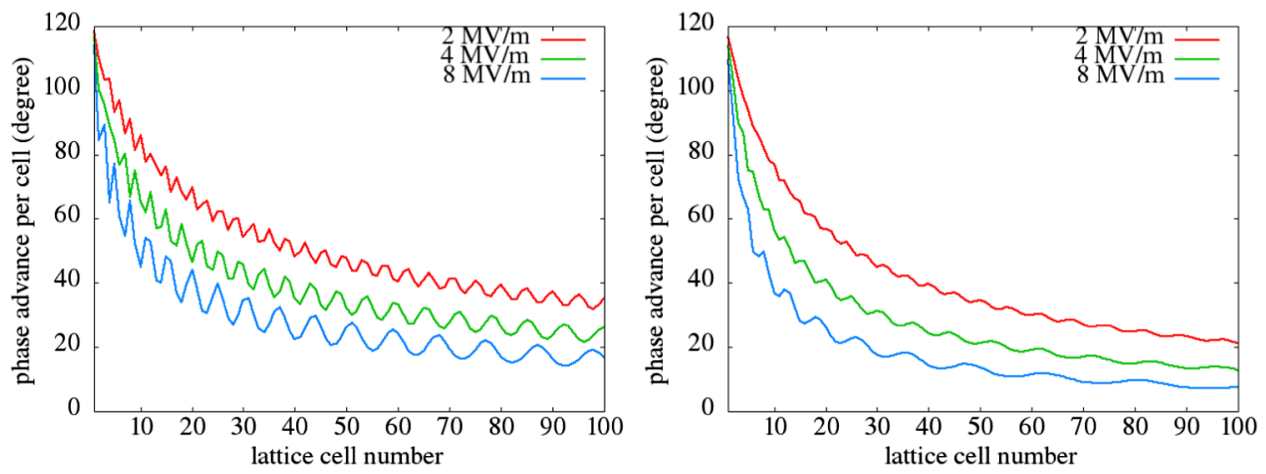

FIG. 6. The zero current horizontal (left) and longitudinal (right) phase advances as a function of lattice cell number in the solenoid-rf channel.
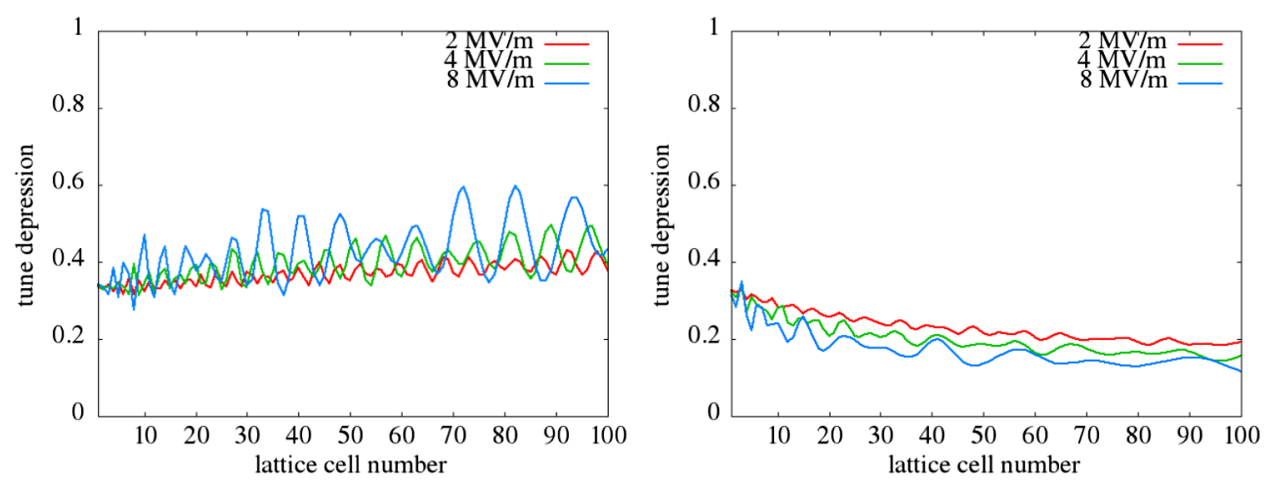

FIG. 7. The horizontal (left) and longitudinal (right) tune depression ratios as a function of lattice cell number in the solenoid-rf channel.

the final emittance growth as a function of accelerating gradient and depressed transverse phase advance in Figs. 4 and 5. Here, the depressed phase advances are from 40 to 75 degrees in both the transverse direction and the longitudinal direction. This range covers the original instability stopband (no acceleration) with large envelope mode growth rate amplitude. Without acceleration or the accelerating gradient is small, the maximum envelope amplitudes are significantly greater than the initial matched rms sizes due to the envelope instability. Most large amplitudes are located around the left corner of the above plot with 40 degree depressed transverse phase advances and small accelerating gradient. This is consistent with the large envelope mode growth rate in the above envelope instability stopband around 40 degree phase advances. With the increase of accelerating gradient, the normalized 
maximum amplitudes decrease and approach the initial rms sizes. The mitigation of the instability is also seen in the final emittance growth. Most emittance growth occur with accelerating gradient below $1 \mathrm{MV} / \mathrm{m}$ due to the instability. With the increase of accelerating gradient, the final emittance growth become smaller and approach a few percent level.

The acceleration mitigates the envelope instability across the stopbands of depressed phase advance. The acceleration inside the rf cavity provides damping to the envelope oscillation as seen in the envelope equations (3). In addition, both the transverse and the longitudinal focusing strengths depend on the energy of the beam. The spacecharge forces also depend on the beam energy. When the beam energy increases, the external focusing strengths and the space-charge effects become weaker. Figure 6 shows the zero current horizontal and longitudinal phase advances per lattice period as a function of the lattice period (cell) number with $2 \mathrm{MV} / \mathrm{m}, 4 \mathrm{MV} / \mathrm{m}$, and $8 \mathrm{MV} / \mathrm{m}$ accelerating gradients. It is seen that with the acceleration, the zero current phase advance quickly falls below 90 degrees after a few lattice periods. This moves the proton beam out of the instability. As the beam energy further increases, the spacecharge forces also become weaker. However, the relative space-charge effects (measured by tune depression ratio) might still be significant. Figure 7 shows the horizontal and the longitudinal tune depression ratios as a function of the lattice cell number using a beam current corresponding to initial 40 degree depressed transverse phase advance without acceleration. Through the entire 100 lattice periods, the horizontal tune depression ratio increases from 0.33 to about 0.4 , while the longitudinal depression ratio decreases from 0.33 to about 0.2 . The relatively strong space-charge effects through the lattice cause the remaining emittance growth as seen in Fig. 3 even with the presence of fast acceleration.

\section{MITIGATION OF THE ENVELOPE INSTABILITY IN A QUADRUPOLE AND RF CHANNEL}

Next, we studied the effects of acceleration on the envelope instability in a transverse quadrupole focusing and longitudinal rf focusing channel using the same proton beam and computational settings. A schematic plot of this periodic channel is shown in Fig. 8. Each period of the lattice consists of a 0.1 meter focusing quadrupole,

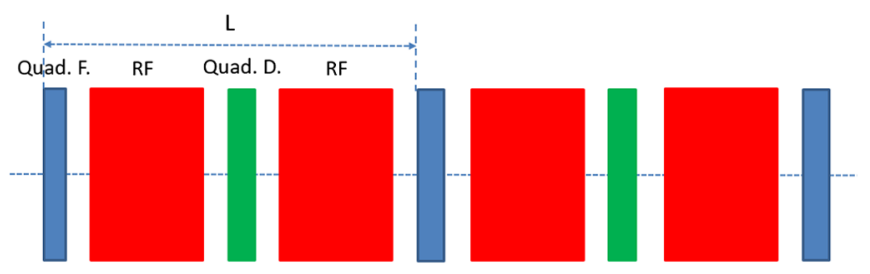

FIG. 8. Schematic plot of a periodic quadrupole and rf channel. a 0.4 meter rf cavity, a 0.1 meter defocusing quadrupole, and another 0.4 meter rf cavity. The total length of the period is 1.4 meters. Figure 9 shows the envelope mode growth rate amplitudes as a function of transverse depressed phase advance inside the above lattice with 120 degree zero current transverse and longitudinal phase advances without and with $8 \mathrm{MV} / \mathrm{m}$ accelerating gradient inside the rf cavities. Without acceleration, the beam shows strong envelope instability. Below about 80 degree phase advances, the envelope mode growth rate amplitude is greater than one and becomes larger as the depressed phase advance decreases with stronger space-charge effects. This instability stopband is caused by the confluent resonance of two envelope oscillation modes. With the presence of acceleration, the envelope instability stopband shrinks significantly. Both the stopband width and the growth rate amplitude become much smaller in comparison to those without acceleration.

We also carried out self-consistent macroparticle simulations using the IMPACT code for this lattice. We first selected a point inside the instability stopband with 40 degree depressed transverse phase advances. Figure 10 shows the horizontal and longitudinal normalized rms size evolution and emittance growth evolution through the quadrupole-rf channel without and with $8 \mathrm{MV} / \mathrm{m}$ accelerating gradient inside the rf cavities. Without acceleration, both horizontal and longitudinal envelope oscillations are unstable and grow up to about 20 periods before reaching saturation. With the acceleration, except the initial small growth in horizontal plane, the rms sizes decrease and become stable through this lattice. The damping of the longitudinal envelope instability due to acceleration is faster than that of the transverse envelope instability. This could be due to a factor of three larger damping rate in the longitudinal envelope equation in comparison to those in the transverse envelope equations. Without acceleration, the normalized emittances increase by more than a factor of 5 due to the envelope instability. With the acceleration, the longitudinal emittance growth is below $30 \%$ and the horizontal growth is below $100 \%$.

Next, we scanned the accelerating gradient inside the rf cavities with depressed transverse phase advance inside the above envelope instability stopband to systematically study the effects of acceleration. Here, the depressed phase advances are from 40 to 70 degrees in the transverse direction and from 48 to about 77 degrees in the longitudinal direction. This range corresponds to large envelope mode growth rate amplitude in the original instability stopband without acceleration. Figures 11 and 12 shows the maximum horizontal and longitudinal rms amplitudes (normalized by the matched rms sizes) within 100 lattice periods and the final emittance growth as a function of accelerating gradient and depressed transverse phase advance. With small or zero accelerating gradient, the maximum amplitudes are significantly greater than the matched envelope sizes due to the envelope instability. 

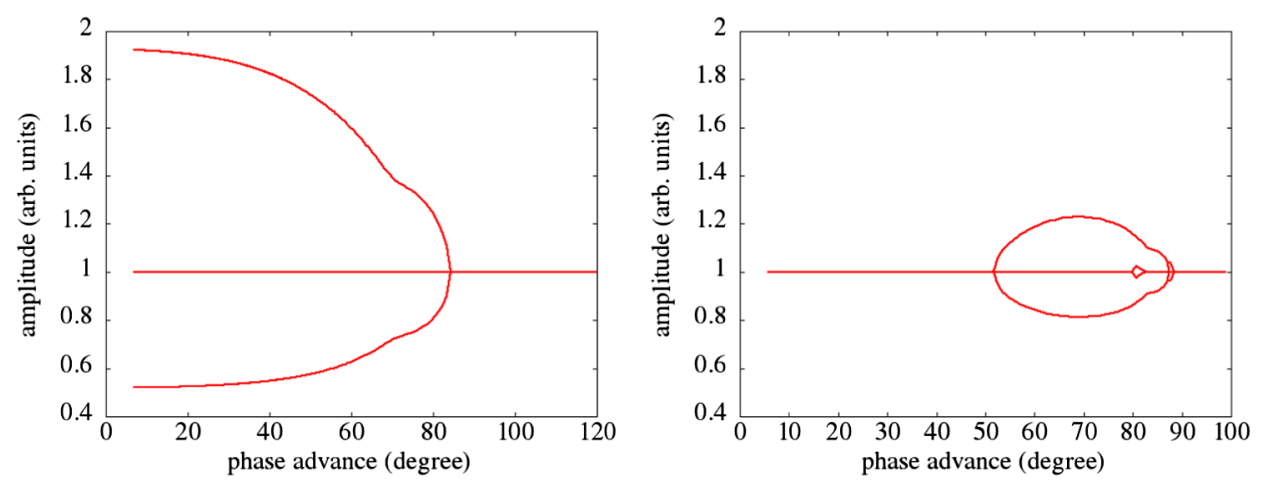

FIG. 9. The 3D envelope mode growth rate amplitudes as a function of depressed transverse phase advance with 120 zero current transverse and longitudinal phase advances in a periodic quadrupole-rf channel without acceleration (left) and with $8 \mathrm{MV} / \mathrm{m}$ accelerating gradient (right).
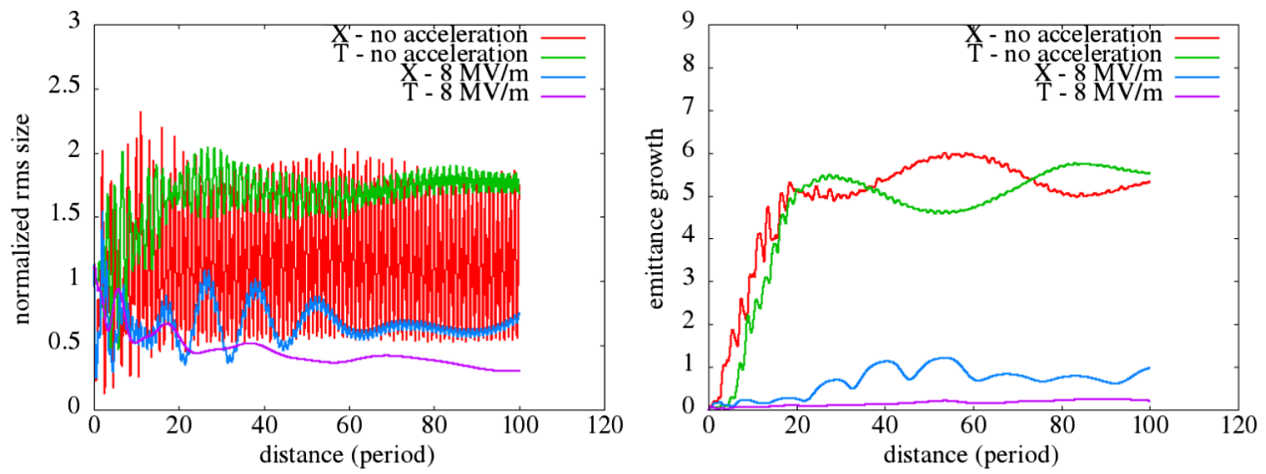

FIG. 10. The horizontal and longitudinal normalized rms size evolution (left) and emittance growth evolution (right) inside the quadrupole-rf channel without acceleration and with acceleration. The depressed transverse phase advance is 40 degrees with 120 degree zero current transverse and longitudinal phase advances.
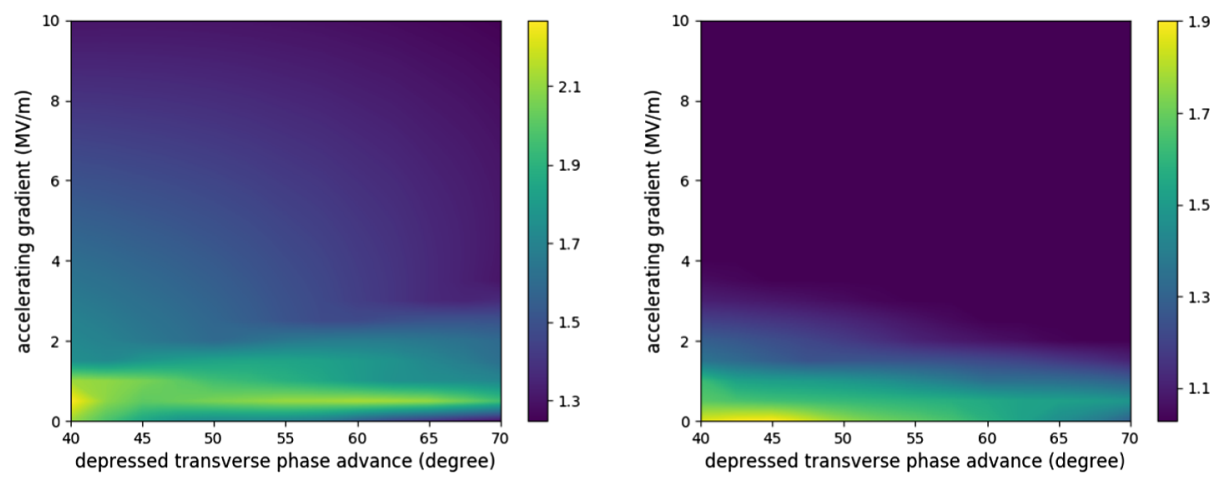

FIG. 11. The maximum normalized horizontal rms amplitude (left) and longitudinal rms amplitude (right) within 100 lattice periods as a function of accelerating gradient and depressed transverse phase advance in the quadrupole-rf channel.

With the increase of accelerating gradient, the maximum amplitudes become smaller and approach the matched envelope sizes. Most large maximum rms amplitudes are located around the left corner of the plot with small depressed transverse phase advance and accelerating gradient. The mitigation of the envelope instability is also seen in the final emittance growth plot. Most emittance growth occur with an accelerating gradient below $2 \mathrm{MV} / \mathrm{m}$. With the increase of the accelerating gradient, the final horizontal and longitudinal emittance growths become smaller and approach the level of $10 \%$. The acceleration helps mitigate the envelope instability across the stopband of depressed phase advance in this lattice too. Figure 13 shows the zero current horizontal and longitudinal phase advances per 

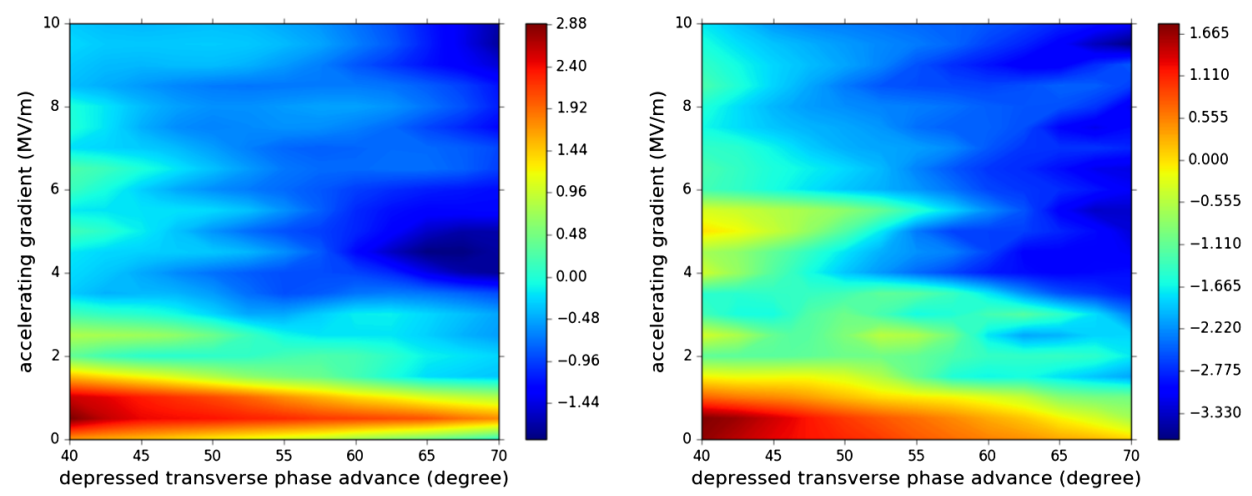

FIG. 12. The final log horizontal emittance growth (left) and longitudinal emittance growth (right) at the end of 100 lattice periods as a function of accelerating gradient and depressed transverse phase advance in the quadrupole-rf channel.
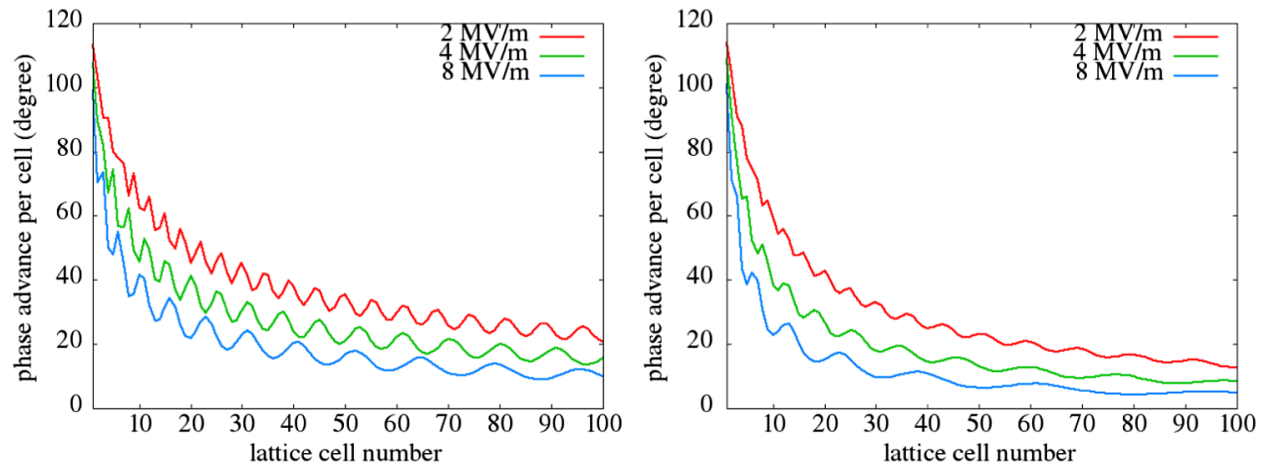

FIG. 13. The zero current horizontal (left) and longitudinal (right) phase advances as a function of lattice cell number in the quadrupole-rf channel.
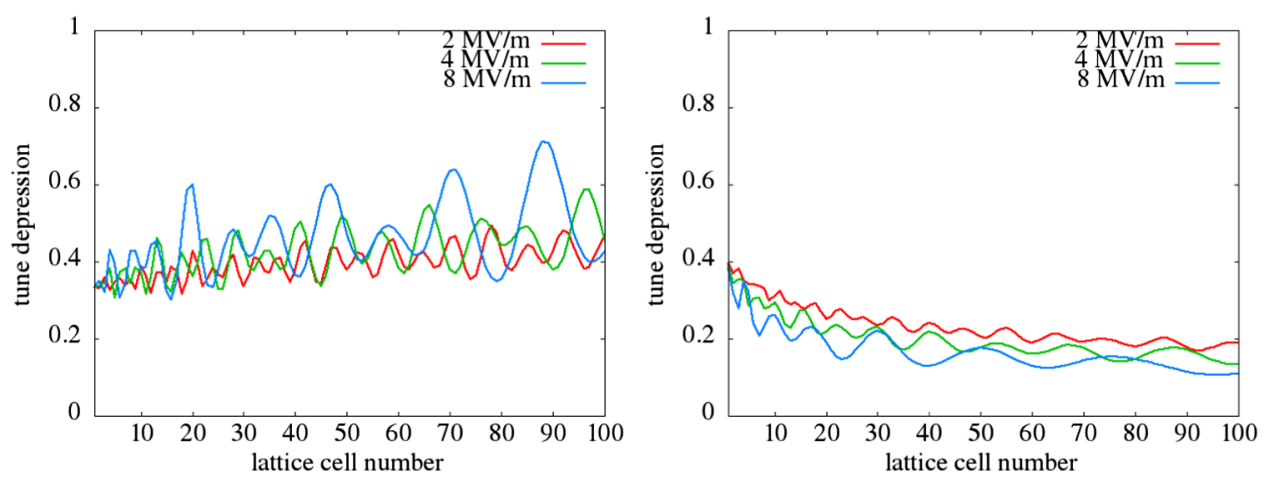

FIG. 14. The horizontal (left) and longitudinal (right) tune depression ratios as a function of lattice cell number in the quadrupole-rf channel.

lattice period as a function of the lattice cell number with $2 \mathrm{MV} / \mathrm{m}, 4 \mathrm{MV} / \mathrm{m}$, and $8 \mathrm{MV} / \mathrm{m}$ accelerating gradients. With the acceleration, the zero current phase advance per lattice period drops below 90 degrees quickly and moves the beam out of the instability.

As the beam energy increases through the accelerating lattice, the space-charge effects become weaker. However, the relative space-charge effects can still be significant. Figure 14 shows the horizontal and longitudinal space-charge tune depression ratios as a function of lattice cell number in the above quadrupole-rf channel with a beam current corresponding to the initial 40 degree depressed phase advance as in Fig. 10. Through the entire lattice, the horizontal tune depression ratio increases from 0.33 to about 0.44 , while the longitudinal tune depression decreases from about 0.4 to about 0.2 . The space-charge effects can still cause some emittance growth even with fast acceleration as seen in Fig. 10. 


\section{CONCLUSIONS}

In this paper, we studied the effects of acceleration on the strong envelope instability using a solenoid-rf lattice and a quadrupole-rf lattice with 120 degree zero current phase advances in both transverse and longitudinal directions. We observed that the beam rms envelope oscillation and final emittance growth from the original envelope instability (no acceleration) were dramatically mitigated due to the rf acceleration in both lattices. The beam moves out of the instability quickly after a few lattice periods due to the acceleration. The envelope oscillation is further damped by the acceleration. This suggests that the 90 degree limit of zero current phase advance per lattice period without acceleration might be violated using fast acceleration in linear accelerators with superconducting rf cavities. This opens additional freedom of choosing transverse and longitudinal focusing parameters in the accelerator design that could result in significant cost savings.

In the above study, we assumed that the external fields were fixed through the entire accelerating lattice. This results in weaker external focusing strengths through the lattice and relatively small space-charge tune depression ratios even though the space-charge effects become weaker with the increase of beam energy. Those relatively strong space-charge effects can still cause some beam emittance growth even with the presence of high accelerating gradient. In the future study, we plan to gradually ramp up the external fields through the lattice to reduce the relative space-charge effects and the beam emittance growth.

\section{ACKNOWLEDGMENTS}

We are grateful for comments by Drs. I. Hofmann and J. Vay and would like to thank Dr. R. D. Ryne for the 3D envelope code. This work was supported by the U.S. Department of Energy under Contract No. DE-AC02$05 \mathrm{CH} 11231$ and used computer resources at the National Energy Research Scientific Computing Center.

[1] I. Hofmann, L. J. Laslett, L. Smith, and I. Haber, Stability of the Kapchinskij-Vladimirskij (K-V) distribution in long periodic transport systems, Part. Accel. 13, 145 (1983).

[2] J. Struckmeier and M. Reiser, Theoretical studies of envelope oscillations and its instabilities of mismatched intense charged-particle beams in periodic focusing channels, Part. Accel. 14, 227 (1984).

[3] G. P. Lawrence and T. P. Wangler, in Proceedings of the Particle Accelerator Conference, Vancouver, BC, Canada, 1997 (IEEE, New York, 1997), p. 1156.

[4] J. Stovall, J. H. Billen, S. Nath, H. Takeda, L. M. Young, D. Jeon, K. R. Crandall, and R. Shafer, in Proceedings of the 19th Particle Accelerator Conference, Chicago, IL, 2001 (IEEE, Piscataway, NJ, 2001), p. 446.
[5] Y. Kondo, M. Ikegami, T. Kobayashi, T. Ohkawa, and A. Ueno, Beam dynamics and commissioning of the J-PARC linac, AIP Conf. Proc. 773, 79 (2005).

[6] F. Gerigk et al., Conceptual Design of the SPL II, CERN2006-006, 2006.

[7] P. N. Ostroumov, Physics design of the $8 \mathrm{GeV} \mathrm{H}$-minus linac, New J. Phys. 8, 281 (2006).

[8] Z. Li et al., Physics design of an accelerator for an accelerator-driven subcritical system, Phys. Rev. ST Accel. Beams 16, 080101 (2013).

[9] M. Eshraqi et al., in Proceedings of the IPAC2014, Dresden, Germany, 2014 (JACoW Publishing, Geneva, Switzerland, 2014), p. 3320.

[10] M. Aiba, S. Machida, Y. Mori, and S. Ohnuma, Resonance crossing experiment at a proof of principle fixed field alternating gradient accelerator, Phys. Rev. ST Accel. Beams 9, 084001 (2006).

[11] S. Y. Lee, Fundamental Limit of Nonscaling Fixed-Field Alternating-Gradient Accelerators, Phys. Rev. Lett. 97, 104801 (2006).

[12] S. Y. Lee, G. Franchetti, I. Hofmann, F. Wang, and L. Yang, Emittance growth mechanisms for space-charge dominated beams in fixed field alternating gradient and proton driver rings, New J. Phys. 8, 291 (2006).

[13] G. M. Wang, T. Shaftan, V. Smaluk, Y. Li, and R. Rand, Lossless crossing of a resonance stopband during tune modulation by synchrotron oscillations, New J. Phys. 19, 093010 (2017).

[14] M. G. Tiefenback, Space charge limits on the transport of ion beams in a long alternating gradient system, Ph.D. thesis, U.C. Berkeley, 1986; Lawrence Berkeley Laboratory Report No. LBL-22465, 1986.

[15] C. Chen and R. C. Davidson, Nonlinear resonances and chaotic behavior in a periodically focused intense chargedparticle beam, Phys. Rev. Lett. 72, 2195 (1994).

[16] S. Lee and A. Riabko, Envelope Hamiltonian of an intense charged-particle beam in periodic solenoidal fields, Phys. Rev. E 51, 1609 (1995).

[17] R. Pakter and F. B. Rizzato, Stability of Periodically Focused Intense Particle Beams, Phys. Rev. Lett. 87, 044801 (2001).

[18] A. V. Fedotov and I. Hofmann, Half-integer resonance crossing in high-intensity rings, Phys. Rev. ST Accel. Beams 5, 024202 (2002).

[19] A. V. Fedotov, I. Hofmann, R. L. Gluckstern, and H. Okamoto, Parametric collective resonances and spacecharge limit in high-intensity rings, Phys. Rev. ST Accel. Beams 6, 094201 (2003).

[20] S. M. Lund and B. Bukh, Stability properties of the transverse envelope equations describing intense ion beam transport, Phys. Rev. ST Accel. Beams 7, 024801 (2004).

[21] L. Groening, W. Barth, W. Bayer, G. Clemente, L. Dahl, P. Forck, P. Gerhard, I. Hofmann, M. S. Kaiser, M. Maier, S. Mickat, and T. Milosic, Experimental Evidence of the $90^{\circ}$ Stop Band in the GSI UNILAC, Phys. Rev. Lett. 102, 234801 (2009).

[22] D. Jeon, L. Groening, and G. Franchetti, Fourth order resonance of a high intensity linear accelerator, Phys. Rev. ST Accel. Beams 12, 054204 (2009).

[23] C. Li and Y. L. Zhao, Envelope instability and the fourth order resonance, Phys. Rev. ST Accel. Beams 17, 124202 (2014). 
[24] C. Li and Q. Qin, Space charge induced beam instability in periodic focusing channel, Phys. Plasmas 22, 023108 (2015).

[25] I. Hofmann and O. Boine-Frankenheim, Space-Charge Structural Instabilities and Resonances in High-Intensity Beams, Phys. Rev. Lett. 115, 204802 (2015).

[26] D. Jeon, J. H. Jang, and H. Jin, Interplay of space-charge fourth order resonance and envelope instability, Nucl. Instrum. Methods Phys. Res., Sect. A 832, 43 (2016).

[27] O. Boine-Frankenheim, I. Hofmann, and J. Struckmeier, Parametric sum envelope instability of periodically focused intense beams, Phys. Plasmas 23, 090705 (2016).

[28] I. Hofmann and O. Boine-Frankenheim, Parametric instabilities in 3D periodically focused beams with space charge, Phys. Rev. Accel. Beams 20, 014202 (2017).

[29] K. Ito, H. Okamoto, Y. Tokashiki, and K. Fukushima, Coherent resonance stop bands in alternating gradient beam transport, Phys. Rev. Accel. Beams 20, 064201 (2017).

[30] Y. Yuan, O. Boine-Frankenheim, and I. Hofmann, Modeling of second order space charge driven coherent sum and difference instabilities, Phys. Rev. Accel. Beams 20, 104201 (2017).
[31] I. Hofmann and O. Boine-Frankenheim, Revisiting the Longitudinal $90^{\circ}$ Limit in High Intensity Linear Accelerators, Phys. Rev. Lett. 118, 114803 (2017).

[32] I. Hofmann, Space Charge Physics for Particle Accelerators (Springer, New York, 2017).

[33] J. Qiang, Three-dimensional envelope instability in periodic focusing channels, Phys. Rev. Accel. Beams 21, 034201 (2018).

[34] F. J. Sacherer, Possible emittance increase through filamentation due to space charge in continuous beams, IEEE Trans. Nucl. Sci. 18, 1101 (1971).

[35] R. Ryne, Los Alamos Report No. LA-UR-95-391, http:// xxx.lanl.gov/abs/acc-phys/9502001.

[36] T. P. Wangler, RF Linear Accelerators, 2nd ed. (WileyVCH, New York, 2008).

[37] J. Qiang, R. D. Ryne, S. Habib, and V. Decyk, An objectoriented parallel particle-in-cell code for beam dynamics simulation in linear accelerators, J. Comput. Phys. 163, 434 (2000).

[38] J. Qiang, R. D. Ryne, M. Venturini, A. A. Zholents, and I. V. Pogorelov, High resolution simulation of beam dynamics in electron linacs for $\mathrm{x}$-ray free electron lasers, Phys. Rev. ST Accel. Beams 12, 100702 (2009). 